\title{
Comparison of blind intubation through supraglottic devices and direct laryngoscopy by novices: a simulation manikin study
}

\author{
Young Yong Kim ${ }^{1}$, Gu Hyun Kang ${ }^{1}$, Won Hee Kim ${ }^{1}$, Hyun Young Choi', \\ Yong Soo Jang ${ }^{1}$, Young Jae Lee', Jae Guk Kim', Hyeongtae Kim', \\ Gyoung Yong Kim²
'Department of Emergency Medicine, Kangnam Sacred Heart Hospital, Hallym University College of Medicine, Seoul, Korea \\ ${ }^{2}$ Gyeonggi Fire Service Academy, Yongin, Korea
}

Objective This study aimed to compare intubation performance between blind intubation through supraglottic airway devices and direct laryngoscopy by novices under manikin simulation. We hypothesized that the intubation time by novices using supraglottic airway devices was superior to that with the Macintosh laryngoscope (MCL).

Methods A prospective, randomized crossover study was conducted with 95 participants, to evaluate i-gel, air-0, LMA Fastrach, and MCL devices. Primary outcomes were the intubation time and the success rate for intubation.

Results The i-gel showed the shortest insertion and tube passing time among the four devices; the i-gel and air- 0 also showed the shortest total intubation time (all $P<0.0083$; i-gel vs. air- 0 , $P=0.03$ ). The i-gel and MCL showed the highest cumulative success rate (all $P<0.0083 ; i-g e l$ vs. $M C L, P=0.12)$.

Conclusion Blind intubation through the i-gel showed almost equal intubation performance compared to direct laryngoscopy.

Keywords Airway management; Intubation; Laryngeal masks
elSSN: 2383-4625

Received: 17 February 2016

Revised: 27 March 2016

Accepted: 31 March 2016

Correspondence to: Gu Hyun Kang Department of Emergency Medicine, Kangnam Sacred Heart Hospital, Hallym University College of Medicine, 1 singil-ro, Yeongdeungpo-gu, Seoul 07441, Korea

E-mail: drkang9@hanmail.net

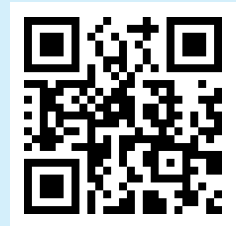

How to cite this article: Kim YY, Kang GH, Kim WH, Choi HY, Jang YS, Lee YJ, Kim JG, Kim H, Kim GY. Comparison of blind intubation through supraglottic devices and direct laryngoscopy by novices: a simulation manikin study. Clin Exp Emerg Med 2016;3(2):75-80.

This is an Open Access article distributed under the terms of the Creative Commons Attribution Non-Commercial License (http:// creativecommons.org/licenses/by-nc/4.0/). 


\section{INTRODUCTION}

Intubation is one of the most important procedures related to prognosis in severely ill patients. ${ }^{1}$ Tracheal intubation is considered the gold standard for protecting the airway. ${ }^{2}$ However, the success rates for intubation are variable depending on airway structure, a patient's clinical status, practitioner's skills, and so forth. ${ }^{3,4}$ The European Resuscitation Council guidelines for cardiopulmonary resuscitation (2010) recommend that intubation be performed by experienced and trained personnel only. ${ }^{5}$ Many health care providers do not have the requisite knowledge and enough practice to perform safe and quick intubation. ${ }^{2}$

For these providers, supraglottic airway devices (SADs) can be a valid alterative. ${ }^{2} S A D$ insertions do not require laryngoscopy and can be used by untrained personnel, or when tracheal intubation is impossible. ${ }^{2}$ SAD has the advantage of easier use without examining the vocal cords in a difficult airway. While tracheal intubation requires effort and time to maintain skills, SADs do not. ${ }^{6}$ However, SADs are limited in their ability to completely maintain and protect the airway. For this reason, blind intubation is required through a SAD after insertion. Many SADs have been developed and many studies have evaluated blind intubation through SADs. LMA Fastrach (Laryngeal Mask Company, San Diego, CA, USA), is one of the SADs developed for tracheal intubation.' It enables intubation, but has a high price, is inconvenient to use, and requires dedicated equipment. SADs can also be used as conduits for fiberoptic bronchoscopy-guided intubation or blind intubation without bronchoscopy. ${ }^{7}$ However, there are few studies of intubation performance using blind intubation through SADs compared to direct Macintosh laryngoscopy (MCL) by novices.

The aim of this study is the evaluation of intubation performance by novices using MCL and SADs under manikin simulation. We hypothesized that the intubation time by novices using SADs was superior to that for MCL.

\section{METHODS}

\section{Study design}

We conducted a randomized, open-label, crossover simulation study to evaluate intubation performance using 3 SADs and MCL. This study was performed at Gyonggi-do fire service academy in May 2015. The local ethics committee approved this study.

\section{Participants}

The sample size was calculated based on a previous study regarding the ventilation time required for intubation. ${ }^{8}$ The intubation time (mean \pm standard deviation, second) in that study was as follows: MCL (36.88 \pm 10.75$)$; Proseal $(43.85 \pm 11.85)$. To detect a 6 -second difference in intubation time using $\alpha=0.05$ and $\beta=0.2$ for an experimental design examining 2 devices, we estimated that at least 50 operators would be required for each device, with a 30\% dropout rate. We recruited 95 trainees (registered nurses and emergency medical technicians) participating in the education program in May 2015. They had no intubation experience clinically, and signed a written consent form before being included.

\section{Equipment and materials}

One direct laryngoscope and 3 SADs were used in this study. A direct laryngoscope is the $M C L$, which has a size 4 curved blade with a Satin Slip Stylet (Mallinckrodt Medical, St. Louis, MO, USA). We also performed intubation using SAD size $4 \mathrm{i}$-gel (Intersurgical, Berkshire, UK), size 3.5 air-0 (Cookgas LLC; Mercury Medical, Clearwater, FL, USA) and size 4 LMA Fastrach. We used a Laerdal airway management trainer (Laerdal, Stavanger, Norway) for performing intubations in this study. A 7.0-mm internal diameter cuffed polyvinyl chloride (PVC) tube (Portex, Keene, NH, USA) was used for blind tracheal intubation by each group. The endotracheal tube (ETT) was lubricated with water-based jelly before insertion.

\section{Interventions}

All participants completed a brief questionnaire consisting of demographic information (age, sex, and licensure) and working experience as a health care provider (Table 1). Before starting the trials, instructors gave lectures for 2 hours about endotracheal intubation (ETI) and SADs. Participants had 50 minutes of practice time with each device to familiarize themselves with all devices and perform intubations on the Laerdal Airway Management Trainer. Ninety-five participants were enrolled, and were randomly allocated to 4 groups: group $A(n=24)$, group $B(n=24)$,

Table 1. Baseline characteristics

\begin{tabular}{lc}
\hline Characteristics & Data $(n=95)$ \\
\hline Sex (\%) & Male $(61.1)$ \\
Age (yr) & $27(25-32)$ \\
Working experience as HCP (yr) & $2.0(0.0-7.0)$ \\
None & $30(31.5)$ \\
$<5$ & $36(37.8)$ \\
$>5$ & $29(30.5)$ \\
License & $17(17.9)$ \\
Registered nurse & $77(81.1)$ \\
1st level EMT & $1(1.1)$ \\
2nd level EMT & \\
\hline Categorical variables are given as number (\%) and continuous variables are given \\
as median (interquartile range). \\
HCP, healthcare provider; EMT, emergency medical technician.
\end{tabular}


group $C(n=24)$, and group $D(n=23)$ (Fig. 1). The random order was generated by a sequence generator (http://www.random.org). After being allocated to a group, the participants performed intubations in sequential order. Independent evaluators were allocated to each device group and used a stopwatch to measure intubation time. They evaluated the success of intubation. All participants performed 3 intubation attempts with each device when allocated to a group. Hence, each participant in each group attempted a total of 12 intubations using 4 devices. Participants had a 10-minute break after 3 intubation attempts for each device. For MCL, placing the manikin's head and neck in a sniffing position was performed by utilizing rolled sheets. Participants inserted the ETT through LMA Fastrach in a reverse orientation, with the concave bend facing posteriorly. For the i-gel, the ETT was inserted by $90^{\circ}$ counterclockwise rotation maneuvres.

\section{Outcomes}

Primary outcomes were the intubation time and the success rate for intubation. The intubation time was measured for 4 sequential procedures by intubators: picking up SAD or $\mathrm{MCL}$, complete insertion of device, successful insertion of ETT, and successful first ventilation.

Time required for SAD insertion (device insertion time, DT) was defined as the time from picking up SAD to complete insertion into the manikin. For $M C L$, DT was alternatively defined as the time from picking up MCL to complete exposure of the larynx. ${ }^{8}$

Time required for ETT insertion (tube insertion time, $\Pi$ ) was defined as the time from picking up SAD or MCL to successful insertion of ETT. Besides MCL, $\Pi$ was measured for the time required to perform blind intubations through SADs.

Time to first ventilation (FVT) was defined as the time from picking up SAD or MCL to successful first ventilation. Successful first ventilation was confirmed by chest rise of the manikin on bagging with a bag-valve mask. Intubation failure was defined as esophageal intubation or exceeding the time limit of 60 seconds.

\section{Statistical analysis}

The data were compiled using a standard spreadsheet application (Excel, Microsoft, Redmond, WA, USA) and were analyzed using the IBM SPSS Statistics ver. 22 (IBM Corp., Armonk, NY, USA). We

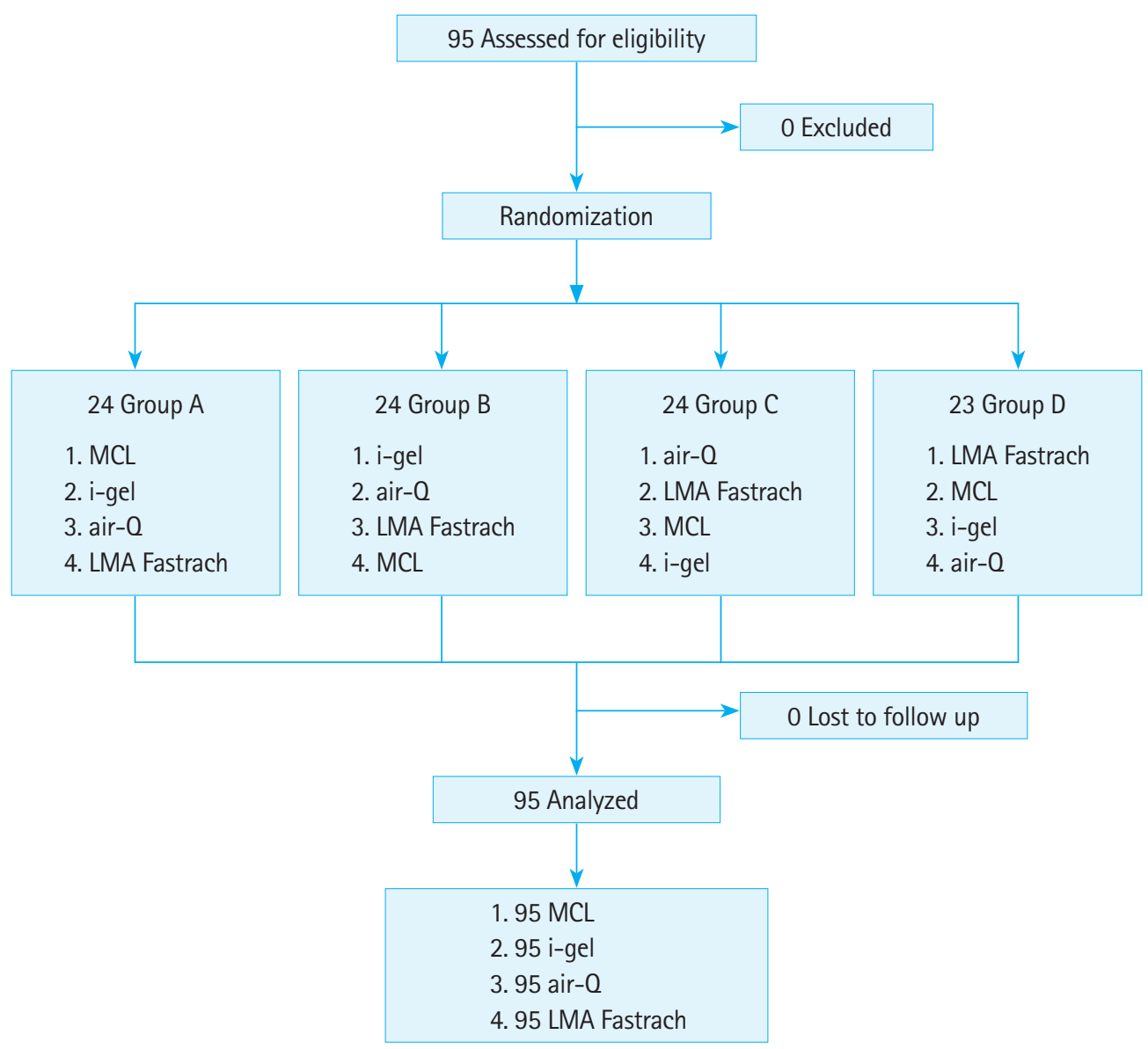

Fig. 1. Flow diagram. MCL, Macintosh laryngoscope. 
generated descriptive statistics and presented them as frequencies and percentages for the categorical data and median with interquartile range for the continuous data. In comparison of the intubation time among the 4 devices, the Friedman test was used for continuous variables. A post-hoc analysis was conducted with the Wilcoxon rank-sum test using the Bonferroni correction. The Kaplan-Meier analysis was performed to analyze the cumulative success rate regarding intubation time. A post-hoc analysis was also performed using the log-rank test with the Bonferroni correction. For all analyzed data, P-value was calculated by two-sided tests and $\mathrm{P}<0.05$ was considered statistically significant. In contrast, in post-hoc analysis, $\mathrm{P}<0.0083$ was considered significant.

\section{RESULTS}

Ninety-five participants were enrolled (Fig. 1). There were no exclusions in our study. The baseline characteristics of the participants are shown in Table 1. DT and $\Pi$ for i-gel were significantly shorter than that for the other 3 devices, whereas DT and $\Pi$ for LMA Fastrach was the longest among the 4 devices (all $P<0.0083$ ) (Table 2). FVT using 3 SADs was the shortest for the i-gel and air-0 (all $P<0.0083$; i-gel vs. air- $0, P=0.03$ ). However, $F V$ for LMA Fastrach was the longest among the 4 devices (all $P<0.0083$ ) (Table 2). TT-DT was the shortest for i-gel among 3 SADs (all $P<$ $0.0083)$, and that for i-gel was not significantly different from $\mathrm{MCL}(\mathrm{P}=0.05)$ (Table 2). FVT-TT was the shortest for air- $\mathrm{Q}$ among 3 SADs (all $P<0.0083$ ) and that for LMA Fastrach was the longest among the 4 devices (all $P<0.0083$ ) (Table 2). The i-gel and MCL showed the highest cumulative success rate (all $P<0.0083$; i-gel vs. $M C L, P=0.12$ ). In contrast, air- $Q$ had the lowest cumu- lative success rates among 4 devices (Fig. 2). The success rate for air- 0 was the lowest among the 4 devices within 3 intubation attempts. Air- $Q$ showed persistently low success rates, even when the number of intubation attempts increased (Fig. 3).
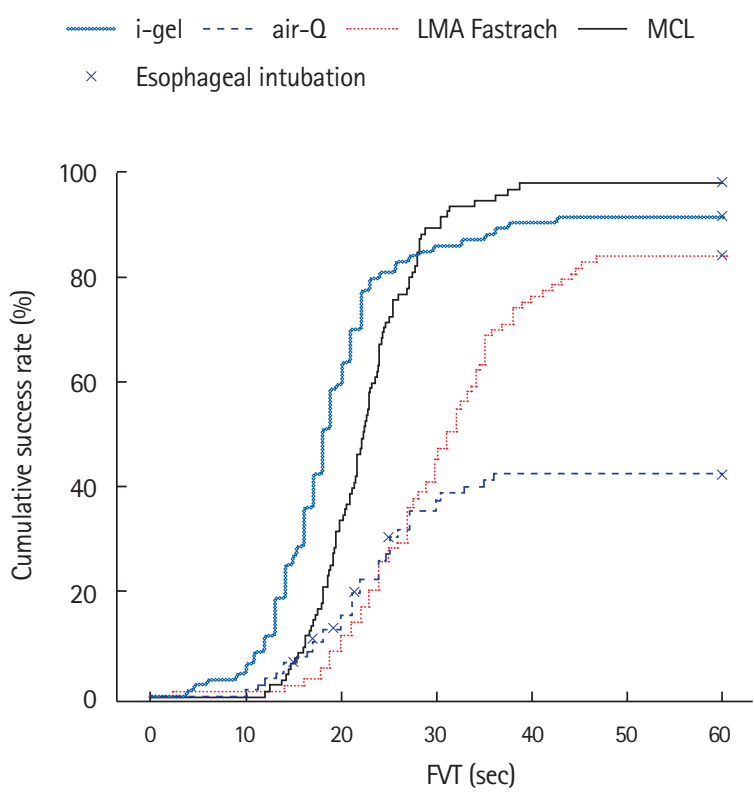

\begin{tabular}{ccccccc}
\hline i-gel & i-gel & i-gel & air-0 & air-0 & LMA \\
vs. & vs. & vs. & vs. & vs. & Fastrach \\
air-0 & LMA Fastrach & MCL & LMA Fastrach & MCL & vs. \\
P-value & $<0.001$ & $<0.001$ & 0.12 & $<0.001$ & $<0.001$ & $<0.001$ \\
\hline
\end{tabular}

Fig. 2. Cumulative success rate of first ventilation time (FVT). Only the intubation time required for first intubation attempt was used for the analysis. MCL, Macintosh laryngoscope. ${ }^{\text {a) }}$ Calculated by Log-rank test with Bonferroni correction. $\mathrm{P}<0.0083$ is considered significant.

Table 2. Comparison of intubation time

\begin{tabular}{|c|c|c|c|c|c|}
\hline & DT (sec) & $\Pi$ (sec) & FVT (sec) & TT-DT (sec) & FVT-TT (sec) \\
\hline i-gel & $3.1(2.3-4.9)$ & $10.5(8.0-14.0)$ & $17.2(13.2-21.0)$ & $7.0(4.0-9.8)$ & $6.3(3.0-8.0)$ \\
\hline Air-0 & $5.0(3.1-7.0)$ & $15.0(10.0-19.2)$ & $15.8(10.2-24.0)$ & $9.6(6.0-13.0)$ & $3.9(2.3-7.0)$ \\
\hline LMA Fastrach & $8.0(5.0-10.0)$ & $20.0(14.0-26.0)$ & $27.6(21.0-34.0)$ & $12.0(6.0-13.0)$ & $7.0(3.9-10.0)$ \\
\hline $\mathrm{MCL}$ & $4.8(3.2-6.5)$ & $12.6(10.3-15.5)$ & $22.0(18.6-25.4)$ & $7.3(5.0-10.0)$ & $9.8(6.9-11.8)$ \\
\hline \multirow[t]{2}{*}{ P-value } & $<0.001$ & $<0.001$ & $<0.001$ & $<0.001$ & $<0.001$ \\
\hline & & & P-value $e^{a)}$ & & \\
\hline i-gel vs. Air-0 & $<0.001$ & $<0.001$ & 0.03 & 0.001 & $<0.001$ \\
\hline i-gel vs. LMA Fastrach & $<0.001$ & $<0.001$ & $<0.001$ & $<0.001$ & 0.354 \\
\hline i-gel vs. MCL & $<0.001$ & 0.002 & $<0.001$ & 0.05 & 0.001 \\
\hline Air- 0 vs. LMA Fastrach & $<0.001$ & $<0.001$ & $<0.001$ & 0.02 & $<0.001$ \\
\hline Air- 0 vs. MCL & 0.73 & 0.1 & $<0.001$ & 0.04 & $<0.001$ \\
\hline LMA Fastrach vs. MCL & $<0.001$ & $<0.001$ & 0.001 & $<0.001$ & 0.007 \\
\hline
\end{tabular}

Continuous variables are given as median (interquartile range). The intubation time from first intubation attempt only is used for analysis. P-value was calculated by Friedman test and $\mathrm{P}<0.05$ is considered significant.

DT, device insertion time; $\Pi$, tube insertion time; FVT, first ventilation time; MCL, Macintosh laryngoscope.

${ }^{a)}$ Calculated by Wilcoxon rank-sum test with Bonferroni correction in post hoc analysis. $\mathrm{P}<0.0083$ is considered significant. 


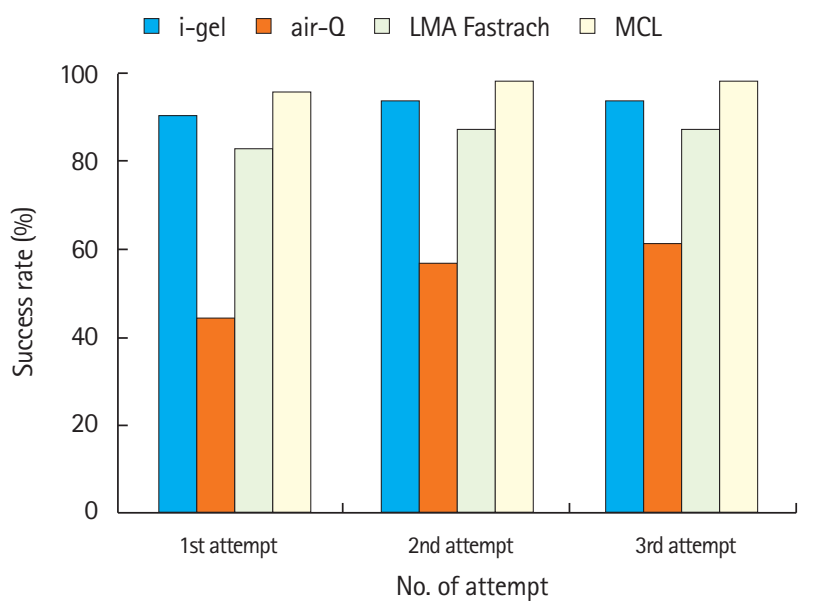

Fig. 3. Comparison of success rate according to the number of intubation attempts. MCL, Macintosh laryngoscope.

\section{DISCUSSION}

It is usually difficult to perform intubation in prehospital emergency care because of patient position and lack of sufficient support for ETI. Training and maintenance of ETI skills in novice health care providers is also expensive and time-consuming. ${ }^{6}$ Studies on ETI and SADs have been performed for prehospital emergency care. ${ }^{6,9,10}$ Based on these studies, SADs are needed for early airway management. Tracheal intubation through SADs is also required in prehospital emergency care.

In this study, i-gel DT and TT-DT were shortest (Table 2). These results were the same as in previous studies (Table 2). ${ }^{7.11}$ Because i-gel is a non-inflation-cuff device, it could be inserted easier and faster than an inflation-cuff device such as a LMA Fastrach. ${ }^{7}$ In contrast, as in a previous study, we found that LMA Fastrach took the longest time for DT. ${ }^{12}$ The insertion time was prolonged because of the rigid structure of the devices during insertion, and because inflation of the cuff requires more time. ${ }^{7}$ One study reported that LMA Fastrach showed shorter tube passing time than air- $0 .{ }^{12}$ This may be due to use of a specific tube for LMA Fastrach that is wire-reinforced, and a soft-tip PVC tube is not standard. We considered that the use of a non-flexible PVC tube prolonged the tube passing time for LMA Fastrach in our study. 13,14

For this study, we chose the SAD insertion maneuvres that showed the highest success rate in previous studies. ${ }^{11,15-17}$ Additionally, PVC ETT was used for all 4 devices. For cumulative success rate, i-gel was the highest among 3 SADs and not significantly different from MCL (Fig. 2). In contrast, the success rate of blind intubation through i-gel was lower than that for LMA Fastrach in previous studies, as compared with those of our study. ${ }^{711,18,19}$
However, we considered whether the difference was related to the intubation experience of the participants or type of tracheal tube, and whether the manikin or patients had an effect on the results. In previous studies, experts in intubation rather than novices performed intubation on patients or manikins. ${ }^{711,18,19}$ Although they were not used to blind intubation through i-gel or LMA Fastrach, there was the consideration of adaptation to new equipment (SADs) because of proficiency with intubation. We also surmised novices preferred the easy teshnique and simplicity of i-gel, compared to LMA Fastrach, unlike the experts in intubation. ${ }^{20}$ These characteristics of i-gel might result in better intubation performance in a novice. A study by Theiler et al. used a reinforced 7.0 $\mathrm{mm}$ single-use ILMA tracheal tube, as compared to our study using a PVC tube. ${ }^{19}$ We considered that the success rate of blind tracheal intubation using LMA Fastrach was influenced by the type of tube used, and a PVC tube through LMA Fastrach resulted in a lower success rate for blind intubation in our study. ${ }^{21}$

Both i-gel and air- $\mathrm{Q}$ were faster than other devices for $\mathrm{FVT}$ (Table 2). We assumed that either the short TT-DT for i-gel or the short FVT-T for air- $\mathrm{Q}$ resulted in an overall decrease of FVT. The success rate for intubation using i-gel was almost as high as with MCL. Hence, i-gel was felt to show overall good intubation performance. However, air- 0 showed the lowest success rate, with frequent esophageal intubations (Figs. 2, 3). These results were consistent with those of previous studies. ${ }^{12,22}$ The reason was supposedly because the elevation ramp of air- $\mathrm{Q}$ for facilitating intubation was insufficient to guide the ETT toward the laryngeal inlet correctly. ${ }^{23}$

There were several limitations in our study. First, we used an airway manikin to simulate a normal patient airway. Thus, the measured intubation performance in this study could differ from that of actual blind intubation through SADs. Secondly, we only used a PVC ETT in this study. However, it has been reported in several studies that the use of a wire-reinforced ETT improved intubation performance. ${ }^{14,21}$ Therefore, the effect of a wire-reinforced ETT on intubation performances needs to be reevaluated.

In conclusion, blind intubation through i-gel showed almost equal intubation performance compared to direct laryngoscopy. More studies on tracheal intubation through SADs in prehospital emergency care should be conducted.

\section{CONFLICT OF INTEREST}

No potential conflict of interest relevant to this article was reported. 


\section{REFERENCES}

1. Schober P, Krage R, van Groeningen D, Loer SA, Schwarte LA. Inverse intubation in entrapped trauma casualties: a simulator based, randomised cross-over comparison of direct, indirect and video laryngoscopy. Emerg Med J 2014;31:959-63.

2. Robak $\mathrm{O}$, Leonardelli M, Zedtwitz-Liebenstein $\mathrm{K}$, et al. Feasibility and speed of insertion of seven supraglottic airway devices under simulated airway conditions. CJEM 2012;14:330-4.

3. Gellerfors M, Larsson A, Svensen CH, Gryth D. Use of the Airtraq device for airway management in the prehospital setting: a retrospective study. Scand J Trauma Resusc Emerg Med 2014; 22:10.

4. Kilicaslan A, Topal A, Tavlan A, Erol A, Otelcioglu S. Effectiveness of the C-MAC video laryngoscope in the management of unexpected failed intubations. Braz J Anesthesiol 2014;64: 62-5.

5. Nolan JP, Soar J, Zideman DA, et al. European Resuscitation Council guidelines for resuscitation 2010 section 1: executive summary. Resuscitation 2010;81:1219-76.

6. Kajino K, Iwami T, Kitamura T, et al. Comparison of supraglottic airway versus endotracheal intubation for the pre-hospital treatment of out-of-hospital cardiac arrest. Crit Care 2011;15: R236.

7. Kapoor S, Jethava DD, Gupta $P$, Jethava D, Kumar A. Comparison of supraglottic devices i-gel and LMA Fastrach as conduit for endotracheal intubation. Indian J Anaesth 2014;58:397402.

8. Ruetzler K, Roessler B, Potura L, et al. Performance and skill retention of intubation by paramedics using seven different airway devices: a manikin study. Resuscitation 2011;82:593-7.

9. Benoit JL, Gerecht RB, Steuerwald MT, McMullan JT. Endotracheal intubation versus supraglottic airway placement in outof-hospital cardiac arrest: a meta-analysis. Resuscitation 2015; 93:20-6.

10. McMullan J, Gerecht R, Bonomo J, et al. Airway management and out-of-hospital cardiac arrest outcome in the CARES registry. Resuscitation 2014;85:617-22.

11. Halwagi $A E$, Massicotte $N$, Lallo $A$, et al. Tracheal intubation through the I-gel supraglottic airway versus the LMA Fastrach: a randomized controlled trial. Anesth Analg 2012;114:152-6.

12. Karim YM, Swanson DE. Comparison of blind tracheal intubation through the intubating laryngeal mask airway (LMA Fastrach) and the Air-0. Anaesthesia 2011;66:185-90.
13. Shah VR, Bhosale GP, Mehta T, Parikh GP. A comparison of conventional endotracheal tube with silicone wire-reinforced tracheal tube for intubation through intubating laryngeal mask airway. Saudi J Anaesth 2014;8:183-7.

14. Sharma MU, Gombar S, Gombar KK, Singh B, Bhatia N. Endotracheal intubation through the intubating laryngeal mask airway (LMA-Fastrach): a randomized study of LMA- Fastrach $^{\text {TM }}$ wire-reinforced silicone endotracheal tube versus conventional polyvinyl chloride tracheal tube. Indian J Anaesth 2013;57:19-24.

15. Joo HS, Rose DK. The intubating laryngeal mask airway with and without fiberoptic guidance. Anesth Analg 1999;88:662-6.

16. Ye L, Liu J, Wong DT, Zhu T. Effects of tracheal tube orientation on the success of intubation through an intubating laryngeal mask airway: study in Mallampati class 3 or 4 patients. $\mathrm{Br} J$ Anaesth 2009;102:269-72.

17. Lu PP, Yang $C H$, Ho AC, Shyr MH. The intubating LMA: a comparison of insertion techniques with conventional tracheal tubes. Can J Anaesth 2000;47:849-53.

18. Michalek P, Donaldson W, Graham C, Hinds JD. A comparison of the I-gel supraglottic airway as a conduit for tracheal intubation with the intubating laryngeal mask airway: a manikin study. Resuscitation 2010;81:74-7.

19. Theiler L, Kleine-Brueggeney M, Urwyler N, Graf T, Luyet $C_{\text {, }}$ Greif R. Randomized clinical trial of the i-gel and Magill tracheal tube or single-use ILMA and ILMA tracheal tube for blind intubation in anaesthetized patients with a predicted difficult airway. Br J Anaesth 2011;107:243-50.

20. Pournajafian A, Alimian M, Rokhtabnak F, Ghodraty M, Mojri M. Success rate of airway devices insertion: laryngeal mask airway versus supraglottic gel device. Anesth Pain Med 2015;5: e22068.

21. Kanazi GE, El-Khatib M, Nasr VG, et al. A comparison of a silicone wire-reinforced tube with the Parker and polyvinyl chloride tubes for tracheal intubation through an intubating laryngeal mask airway in patients with normal airways undergoing general anesthesia. Anesth Analg 2008;107:994-7.

22. Garzon Sanchez JC, Lopez Correa T, Sastre Rincon JA. Blind tracheal intubation with the air-Q (ILA-Cookgas) mask. A comparison with the ILMA-Fastrach laryngeal intubation mask. Rev Esp Anestesiol Reanim 2014;61:190-5.

23. Cookgas. Features of air- 0 masked laryngeal airways [Internet]. Saint Louis, MO: Cookgas [cited 2015 Aug 30]. Available from: http://cookgas.com/index.php/air-q-disposable-2/. 